\title{
Subtotal perirhinal cortex lesions increase exploratory behavior in the rat without producing deficits in the Morris water maze
}

\author{
KJESTEN A. WIIG and DAVID K. BILKEY \\ University of Otago, Dunedin, New Zealand
}

\begin{abstract}
Rats with bilateral, subtotal lesions of the superficial layers of perirhinal cortex were tested in a reaction-to-novelty procedure and in the Morris water maze. Lesioned rats spent significantly more time than did control animals in exploring objects in the reaction-to-novelty test apparatus. In addition, lesioned rats exhibited increased rearing, both in the exploration procedure and in an open field test. Locomotor and grooming activity in lesioned rats was not significantly different from that of control animals. These results suggest that, in the rat, the perirhinal cortex may be involved in the modulation of exploratory behavior, possibly via links with the amygdala. Lesioned rats were not impaired in the Morris water maze; rather, their latencies to find the hidden platform were significantly lower than those of control animals during initial place navigation testing. The latter finding contrasts with the results of our previous work, which demonstrates that more extensive lesions of perirhinal cortex produce water maze deficits.
\end{abstract}

In recent years, researchers have become aware that the function of the hippocampus is intimately related to that of the surrounding parahippocampal region, which, in the rat, incorporates the entorhinal, perirhinal, and pre- and parasubicular cortices (Squire, 1992; Witter, Groenewegen, Lopes da Silva, \& Lohman, 1989). Anatomical evidence suggests that these regions are closely associated, as in both the monkey and the rat the perirhinal cortex provides a major portion of the cortical input to the entorhinal cortex (Deacon, Eichenbaum, Rosenburg, \& Eckmann, 1983; Insausti, Amaral, \& Cowan, 1987), which in turn is a major extrinsic input to the hippocampal formation (Blackstad, 1958; HjorthSimonsen, 1972). Furthermore, the perirhinal cortex is also the source of a direct projection to hippocampal subfield CA1 and the subicular region (Deacon et al., 1983; Koesel, van Hoesen, \& Rosene, 1983; Suzuki \& Amaral, 1990). The perirhinal cortex is located in the posterior portion of the rhinal sulcus, situated ventral to the auditory cortical areas TE2 and TE3, and dorsal to the entorhinal cortex (Zilles, 1990), and it receives a cascade of multimodel information from a large number of cortical and subcortical regions. Efferents from the orbital, cingulate, insular, temporal, parietal, and occipital cortices project into perirhinal cortex, as well as fibers originating in the subiculum, diagonal band of Broca, nucleus reuins of the thalamus, and several amyg-

K.A.W. was supported by a William Evans scholarship. This research was supported by a grant from the New Zealand Lotteries Grants Board and the Health Research Council of New Zealand to D.K.B. Correspondence should be addressed to D. K. Bilkey, Department of Psychology, University of Otago, P.O. Box 56, Dunedin, New Zealand (e-mail: sycodkb@otago.ac.nz). daloid nuclei (Deacon et al., 1983). In addition, perirhinal cortex efferents have been shown to make reciprocal connections back to many of these areas (Witter et al., 1989). The perirhinal cortex is, therefore, considered to be part of a major pathway through which information is exchanged between cortical and limbic areas (Deacon et al., 1983; Saunders \& Rosene, 1988; Zola-Morgan, Squire, Amaral, \& Suzuki, 1989).

As evidence of functional links between these regions, several behavioral studies have demonstrated that similarities exist between the effects of hippocampal lesions and lesions of the cortical areas that are anatomically related to this structure. For a number of years, it has been known that lesions of the hippocampus or entorhinal cortex result in severe memory disorders (e.g., Morris, Garrud, Rawlins, \& O'Keefe, 1982; Shenck \& Morris, 1985; Squire, 1992). Similarly, it has recently been demonstrated that monkeys with bilateral lesions that include perirhinal cortex show severe deficits on several memory tasks (Murray \& Mishkin, 1986; Suzuki, Zola-Morgan, Squire, \& Amaral, 1993; ZolaMorgan et al., 1989) and that perirhinal lesions exacerbate memory impairment following hippocampal damage (Zola-Morgan, Squire, Clower, \& Rempel, 1993). Rhinal (entorhinal and perirhinal) cortex ablations also result in deficits in a delayed nonmatching-to-sample task in monkeys (Gaffan \& Murray, 1992) and in an olfactory delayed matching-to-sample task in rats (Otto \& Eichenbaum, 1992). Furthermore, near-total bilateral perirhinal cortex lesions produce an impairment in the water maze task in the rat (Wiig \& Bilkey, 1993).

Lesions of the limbic system have also been shown to produce alterations in nonmnemonic tasks. There is evidence, for example, that lesions of the hippocampus, 
the entorhinal cortex, and the amygdala all separately affect aspects of exploratory behavior (Jarrard, 1968; Kohler \& Sundberg, 1977; Myhrer, 1988, 1989). Given the connectivity between perirhinal cortex and both the hippocampus and the entorhinal cortex, it is possible that perirhinal lesions may also have some effect on exploratory behavior. Furthermore, since some evidence suggests that exploratory behavior may in part be mediated through the amygdala (White \& Weingarten, 1976), disruption of the connections from the superficial layers of the perirhinal cortex, which connect reciprocally to the lateral nucleus of the amygdala (Witter et al., 1989) may produce these nonmnemonic effects without producing the classical hippocampal-type deficits in memory or spatial behavior.

In the present experiment, this possibility was investigated by testing animals with bilateral subtotal electrolytic lesions of the superficial perirhinal cortex in an exploratory and reaction-to-novelty task, and in the Morris water maze. Performance in the latter procedure has been shown to be affected by both hippocampal and total perirhinal cortex lesions ( Morris et al., 1982; Wiig \& Bilkey, 1993).

\section{METHOD}

\section{Subjects}

Twenty male Sprague-Dawley rats 3-4 months of age were individually housed in wire mesh cages, where they had free access to food (Rat Blox) and water. The animals were maintained on a 12:12-h light:dark cycle, at a constant temperature of $23^{\circ} \mathrm{C}$. All surgical and behavioral procedures were conducted during the light cycle.

\section{Surgical Procedures}

The animals were randomly assigned to two groups. One group ( $n=10)$ served as sham operated controls, and the second group $(n=10)$ received subtotal bilateral perirhinal cortical lesions. All animals were anesthetized with sodium pentobarbital $(50 \mathrm{mg} / \mathrm{kg}$, i.p.) and were placed in a stereotaxic apparatus. A midline incision was made, the scalp retracted, and holes drilled at coordinates 6.3 and $7.0 \mathrm{~mm}$ posterior to bregma and $5.4 \mathrm{~mm}$ lateral to the midline. In 5 control animals, the wound was then sutured and the animals recovered from anesthesia. In the remaining animals, bipolar electrodes (two twisted Teflon coated wires, $125 \mu \mathrm{m}$ in diameter) were lowered through the trephines at an angle of $10^{\circ}-12^{\circ}$ from the vertical in the sagittal plane and $6.5 \mathrm{~mm}$ ventral measured from the surface of the brain, to the perirhinal cortex of each hemisphere. In 5 control animals, the electrodes were then withdrawn, whereas in the 10 animals from the lesion group, direct current at $50 \mu \mathrm{A}$ was passed through the electrodes for $8 \mathrm{sec}$. Following the completion of the surgery, the wound was sutured and the animals were kept warm and monitored until spontaneous movement occurred. Once the animals were stabilized, they were returned to their home cages and left to recover for 12 days prior to behavioral testing.

\section{Behavioral Procedures}

Exploration and reaction to novelty. The experimental chamber consisted of a $60 \times 60 \mathrm{~cm}$ hardboard box with walls $20 \mathrm{~cm}$ high. Three of the walls were made of hardboard, and the fourth consisted of a clear Plexiglas panel. The floor of the box was divided into 36 equal-sized squares. The box was elevated $1 \mathrm{~m}$ above the floor and was illuminated from above by a single in- candescent light bulb. At various times during the experiment, objects were placed into the box. Three of these objects (familiar stimuli) consisted of identically sized cubes of wood, measuring $4 \times 3 \mathrm{~cm}$. The other four objects (novel stimuli) were small multicolored childrens' toys. Behavior was coded by an observer who used a computerized event timer that simultaneously recorded the frequency and duration of up to four different behaviors. Trials were also videotaped, and a subsample was analyzed by an observer blind to the animals' condition. The reliability of scoring between observers was then calculated. Each day's training involved two 5-min sessions. In the first session (Phase 1), each rat was placed in the experimental chamber with the three "familiar" objects. The amount of time spent grooming, number of squares traversed, amount of time and frequency of contact with each object, and amount of time and frequency of wall-supported rearing were recorded. After the 5-min period had elapsed, each rat was removed from the apparatus and was returned to a holding box where it remained for $20 \mathrm{~min}$. In the second session (Phase 2), each rat was placed in the box with two of the familiar objects and one of the novel objects. During this period, the behaviors were recorded as for Phase 1. The whole procedure was repeated for 4 consecutive days, with a different novel object used on each day. The novel objects were randomly moved to a new position from trial to trial, to exclude the possibility of positional effects. The apparatus and objects were cleaned and dried between each trial.

Rearing in an open field. Rearing was also separately assessed for a subset of 12 animals (6 control and 6 lesioned rats) on an open table top. The table was located in a different room to that used above and was illuminated by a single incandescent light bulb. The rats were individually placed on the table top for one 10-min session, during which the number of rears was recorded.

Morris water maze. The test apparatus consisted of a circular pool, measuring $150 \mathrm{~cm}$ in diameter. The pool was filled with water to a depth of $29 \mathrm{~cm}$ and was maintained at a constant temperature of $27^{\circ} \mathrm{C}$. Four points around the edge of the pool were arbitrarily designated as north, south, east, and west, which allowed the pool to be divided into four corresponding quadrants (e.g., northeast, southeast, southwest, northwest). The pool contained a clear Perspex platform $(10 \times 10 \mathrm{~cm})$ that was adjustable in height. The platform could be raised or lowered so that it rested $1 \mathrm{~cm}$ above or below the water level. In the cued navigation condition, an orange circular sponge measuring $7.5 \mathrm{~cm}$ in diameter was attached to the top of the platform. Extramaze cues, which consisted of animal cages and laboratory furniture, were held constant throughout the experiment.

At the start of each trial, a rat was placed in the water facing the wall of the pool. The starting point (north, south, east, or west) was randomly determined, and varied on every trial. The rat was then allowed to swim in the pool until it located the platform, where it was permitted to remain for $10 \mathrm{sec}$ before being removed and placed in a holding box. If the rat did not find the platform within 2 min after being placed in the pool, it was immediately removed and returned to the holding box. There was a 2 -min interval between trials.

Testing was conducted on 10 consecutive days, at the same time each day (1800-2200 h), with eight trials per animal per day. The procedure consisted of three conditions, in the following order: place navigation (PN1) for 4 consecutive days, cued navigation (CN) on days 5-7, and place navigation (PN2) on Days 8-10. During PN1, the platform was submerged $10 \mathrm{~mm}$ below the water line and located in the middle of the northwest quadrant of the pool. Following the completion of PN1, a probe trial was conducted during which the platform was removed from the pool and the animal was allowed to swim freely for $1 \mathrm{~min}$ before being removed. During the $\mathrm{CN}$ trials, the platform was moved to the middle of the southeast quadrant and was raised so that it rested $10 \mathrm{~mm}$ above the water level. The orange circular cue was at- 
tached to the top of the platform. A second probe trial was conducted following the last trial of $\mathrm{CN}$ (Day 7), during which the platform was once again removed from the pool. For PN2, the cue was removed and the platform (still in the SE quadrant) was lowered so that it rested $10 \mathrm{~mm}$ below the level of the water. A final probe trial followed the completion of this phase of the experiment.

All trials were videotaped with a video camera positioned directly above the pool. Following the completion of testing, the video tapes were replayed for data analysis. For each trial, the time to reach the platform (escape latencies), swim speed, and swim distance were recorded. Probe trials were analyzed to determine both the number of annulus crossings and the amount of time that each animal spent in each of the four quadrants.

\section{Histological Procedures}

On completion of the behavioral experiments, the animals were euthenized with an overdose of sodium pentobarbital and perfused through the heart with saline $(0.9 \%)$, followed by a $10 \%$ formalin solution. The brains were removed and immersed in a sugar-formalin solution (30\%) until sectioning. Each brain was sectioned (40- $\mu \mathrm{m}$ steps), mounted, and stained with thionin. Every second section was then analyzed blind, to obtain a measure of lesion area (compared with the total area of the structure as outlined in Figure 1) for each hemisphere of each lesioned animal. This analysis required the use of a computerized procedure which determined the area within a border specified by the operator. An estimate of the total volume of damaged perirhinal cortex was then made by summing each measure of lesion area and describing this as a percentage of total perirhinal volume as determined by measures made from the same sections.

\section{RESULTS}

\section{Lesion Verification}

The lesion foci were visible under a light microscope as an eliptical area approximately $0.5-0.8 \mathrm{~mm}$ in diameter characterized by neuronal loss and gliosis (see Figure 1). In 7 out of the 10 animals, lesions were limited to the perirhinal cortex, damage being predominantly to the superficial regions of this structure. Two other animals also sustained minor damage to the medial and lateral entorhinal cortices, while a third also had minimal damage to the presubicular area. This extraperirhinal pathology appeared as small clusters of darkly stained cells, with a slight reduction in cell density. No visible damage to anatomically related regions such as the thalamic nuclei, amygdala, diagonal band of Broca, or other cortical areas was detected. The extent of damage to the perirhinal cortex was determined by measuring the area of lesion in each of 15-20 coronal sections in each animal. The mean percentage of perirhinal cortex lesioned was $22.1 \%( \pm 3.4 S E M)$ for the left hemisphere and $28.6 \%( \pm 6.3)$ for the right.

\section{Behavior}

The sham operated and lesion-tract control rats did not differ significantly in any of the following measures and were treated as a single control group. Likewise, the 3 animals that sustained minor damage to entorhinal and presubicular areas did not differ from other lesioned animals on any behavioral analyses, so they were included in the experimental group for statistical analysis. Interobserver agreements of $86 \%$ and $93 \%$ were obtained for the exploration and rearing measures, respectively.

Exploration and reaction to novelty. Figure 2 illustrates the total amount of time spent in exploring the neutral and novel objects by all rats across the 4-day experimental procedure. Both the control and the experimental animals showed a preference for novelty, as measured by time spent exploring the novel versus neutral objects $[F(1,18)=110.78, p<.0001]$. It is apparent, however, that the perirhinal lesioned animals spent significantly more time exploring the novel objects than did the control rats across all 4 days $[F(1,18)=22.66$, $p<.05]$. This appears to be the result of a greater tendency for the lesioned animals, as opposed to the controls, to revisit the novel object $[F(1,18)=74.556, p<$ $.05]$, for there were no significant differences between groups for the mean length of each interaction with the novel object (refer to Table 1).

The perirhinal lesioned animals also spent more time than control animals did in exploring the neutral objects across all 4 days $[F(1,18)=26.244, p<.05$; see Figure 2]. Once again, this was the result of a greater number of visits back to the neutral objects $[F(1,18)=$ $27.564, p<.05$ ], for there were no significant differences between the two groups for the mean length of each interaction with the neutral object (Table 1).

The total time spent in wall rearing per session increased over the 4-day experimental period for both control and lesioned rats $[F(1,18)=22.936, p<.05]$. The perirhinal lesioned animals, however, spent significantly more time in wall rearing than did the control animals, as is illustrated in Figure $3[F(1,18)=58.579$, $p<.05]$. Both the frequency of wall rears and the mean length of each wall rear were found to be significantly greater for the perirhinal lesioned animals than for the control rats $[F(1,18)=8.038, p<.05$, and $F(1,18)=$ 21.762, $p<.05$; see Table 1].

Although the perirhinal lesioned rats tended to spend less time grooming than the control animals did, this difference did not approach statistical significance. Similarly, although there was a tendency for the lesioned animals to display a greater amount of locomotor activity, there was no significant difference in the number of grid squares traversed by each group during the 4-day experimental procedure.

Table 1

Mean Duration and Frequency of Object Exploration and Rearing over the Entire 4-Day Experimental Procedure

\begin{tabular}{|c|c|c|c|c|c|c|}
\hline \multirow[b]{3}{*}{ Group } & \multicolumn{4}{|c|}{ Object } & \multirow{2}{*}{\multicolumn{2}{|c|}{ Rearing }} \\
\hline & \multicolumn{2}{|c|}{ Neutral } & \multicolumn{2}{|c|}{ Novel } & & \\
\hline & $\overline{\text { Dur }}$ & Freq & Dur & Freq & Dur & Freq \\
\hline Control & 2.02 & 8.38 & 1.55 & 6.57 & 6.66 & 31.77 \\
\hline Lesion & 2.11 & $14.8^{*}$ & 2.24 & $12.16^{*}$ & 9.75 & $41.0^{*}$ \\
\hline
\end{tabular}

Note-Dur, duration, given in seconds; Freq, frequency. ${ }^{*}$ Significant difference between control and lesioned groups at $p<.05$. 

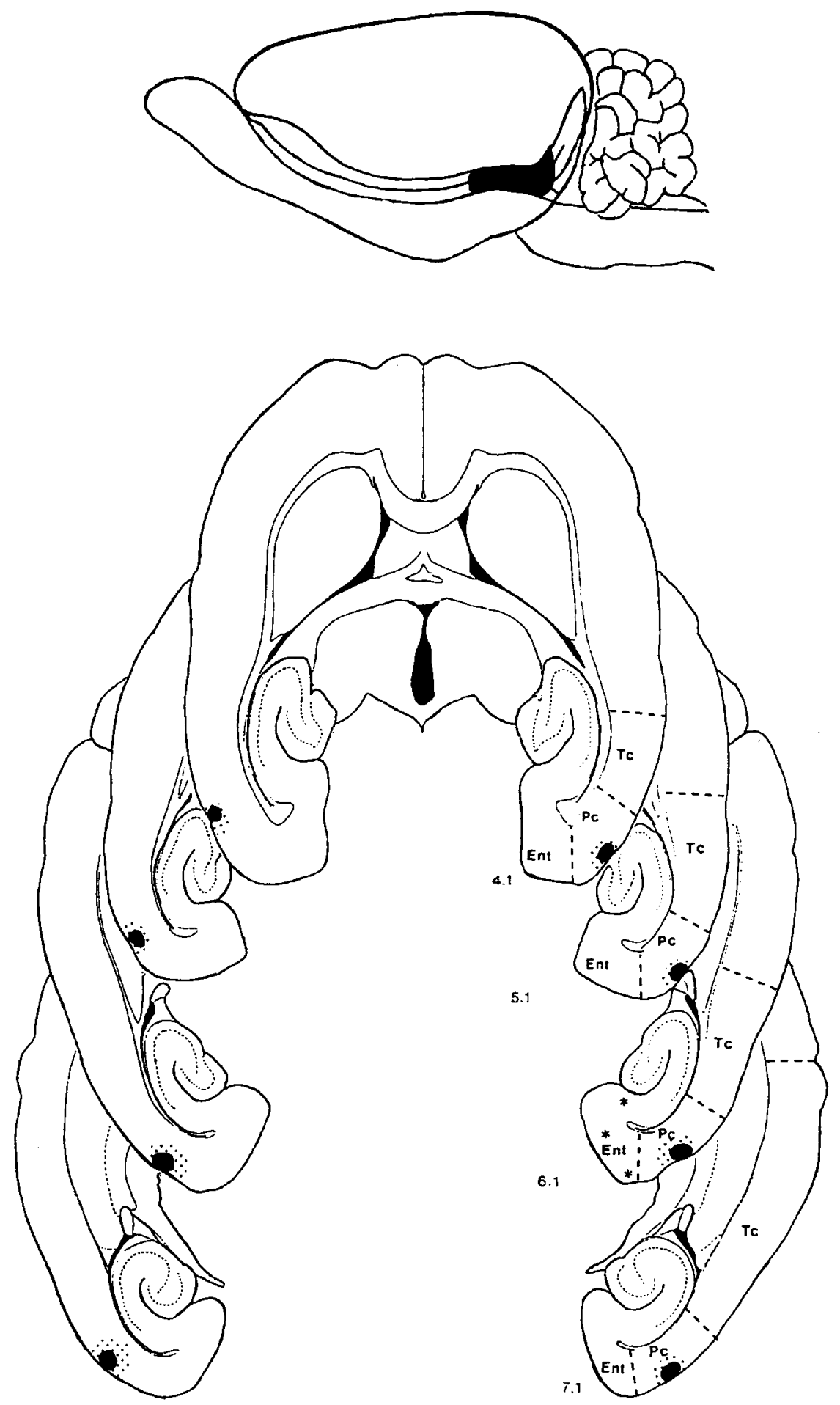

Figure 1. Top: Shaded area represents location of perirhinal cortex in a lateral view of the rat brain. From "Afferent Connections of the Perihinal Cortex in the Rat," by $T$. W. Deacon, H. Eichenbaum, P. Rosenberg, and K. W. Eckmann, 1983, Journal of Comparative Neurology, 220, p. 172 (Figure 3). Copyright 1983 by John Wiley \& Sons, Inc. Adapted by permission. Bottom: Horizontal section through rat brain illustrating the minimum (dark) and maximum (stippled) extent of perirhinal cortex lesions. Pc, perirhinal cortex; Ent, entorhinal cortex; Tc, temporal cortex. Numbers represent the vertical distance from bregma; asterisks mark the location of minor damage to extraperirhinal regions. From The Rat Brain in Stereotaxic Coordinates (Figures 57, 59, 61, and 63), by G. Paxinos and C. Watson, 1982, Sydney: Academic Press. Copyright 1982 by Academic Press. Adapted by permission of Harcourt Brace Jovanovich. 

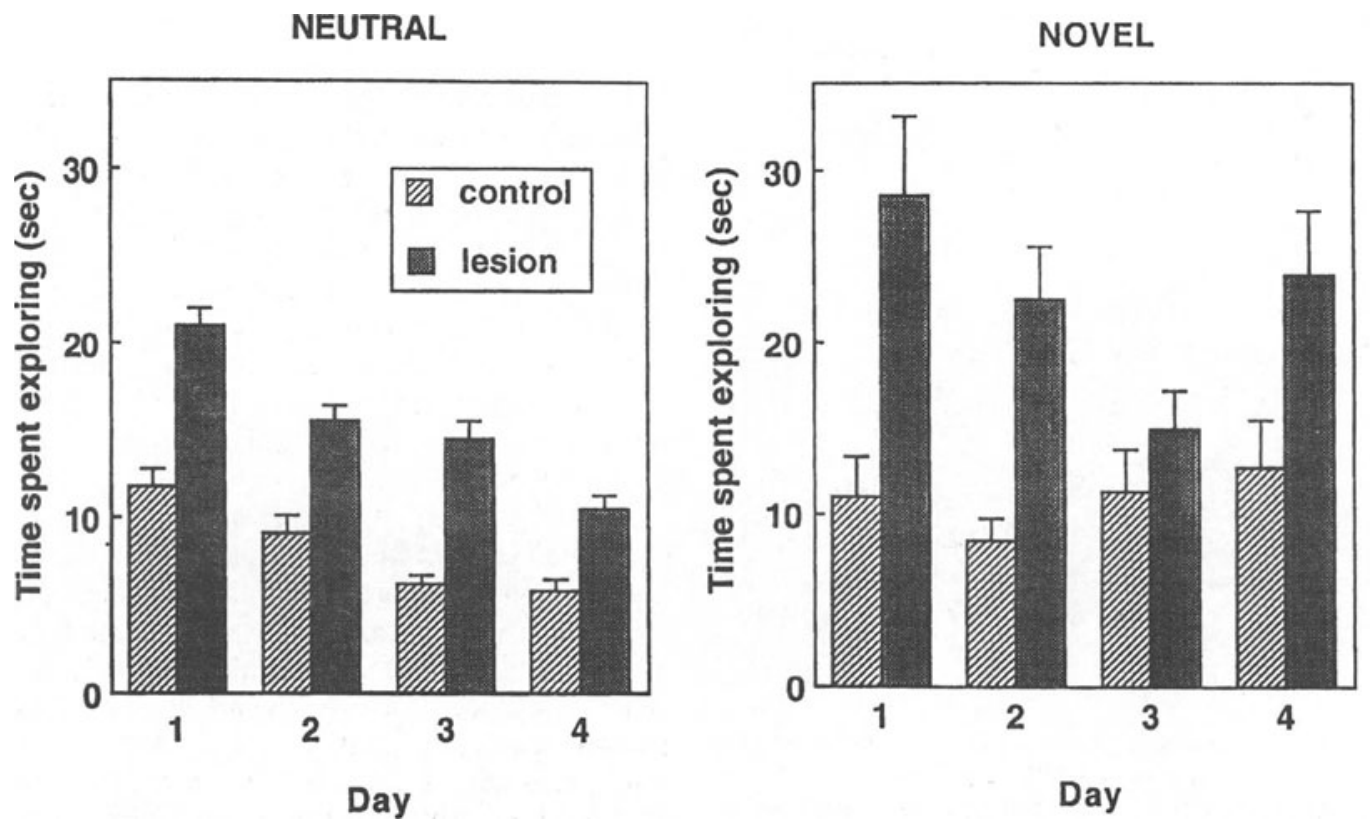

Figure 2. Mean time per day spent exploring the neutral and novel stimuli by control and lesioned animals dur-
ing Phase 2 of the reaction-to-novelty procedure. Perirhinal lesioned rats spent significantly more time than did
controls in exploring both types of stimuli. Error bars indicate $S E M$.

Rearing in the open field. The numbers of rears a subset of control $(n=6)$ and experimental $(n=6)$ animals made over a 10 -min period were analyzed. It was found that the perirhinal lesioned animals made significantly more rears than the control animals did over the $10-\min$ period $[F(1,109)=7.30, p<.01]$.

Place Navigation 1 in the water maze. An analysis of escape latencies during $\mathrm{PN} 1$ revealed that both

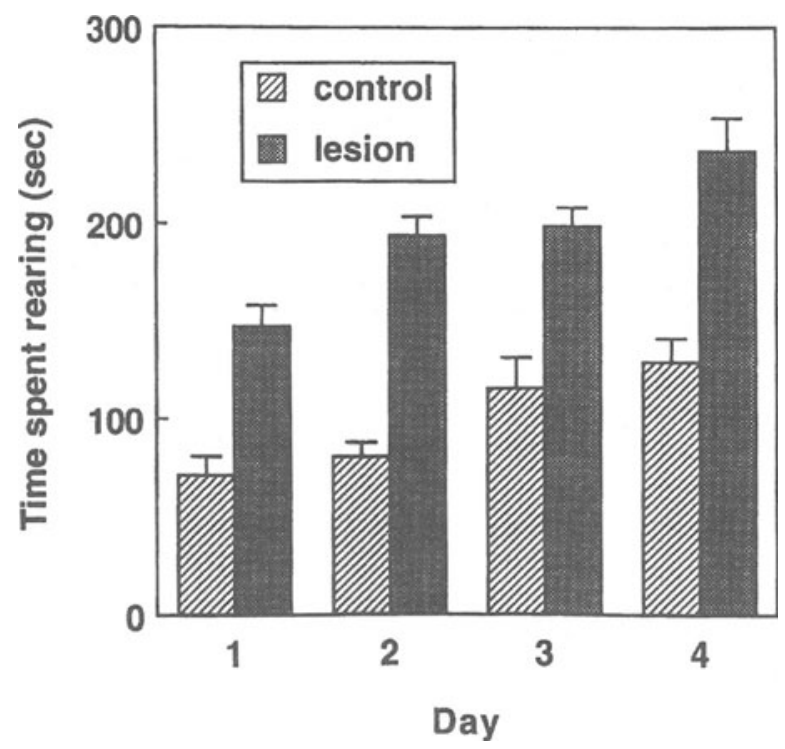

Figure 3. Mean time spent in wall-supported rearing during the 10-min reaction-to-novelty procedure. Lesioned animals spent significantly more time rearing than did control animals. groups of animals showed a decrease in the time taken to reach the platform as trials progressed $[F(3,72)=$ $48.085, p<.001]$ (see Figure 4). Interestingly, perirhinal lesioned animals had significantly shorter latencies for escaping than did the control animals $[F(1,72)=$ $9.381, p<.01]$. Repeated measures analyses of variance (ANOVAs) performed separately on swim speed and swim distance for control and lesioned animals revealed that there were no significant differences on either measure.

An ANOVA performed on the time spent in each of the four quadrants during the probe trial revealed a significant difference $[F(3,75)=67.52, p<.0001]$. A subsequent post hoc analysis (Student-Newman-Keuls) demonstrated that both groups of animals spent a greater amount of time in the quadrant that had formerly contained the escape platform than would have been expected by chance $(p<.05)$. There were, however, no significant differences between groups. An unpaired $t$ test revealed that there were also no significant differences in the number of platform crossings made by control and lesioned animals.

Cued navigation in the water maze. In the cued navigation phase of the experiment, both groups of animals showed a significant decrease in escape latency as trials progressed $[F(2,54)=42.713, p<.0001]$, but with no significant differences between groups. An ANOVA performed on the amount of time that the control and lesioned rats spent swimming in each of the four quadrants during the probe trial demonstrated that there were no significant differences between groups, but there was a significant effect of quadrant $[F(3,75)=$ $51.89, p<.0001]$. Post hoc analysis revealed that both 


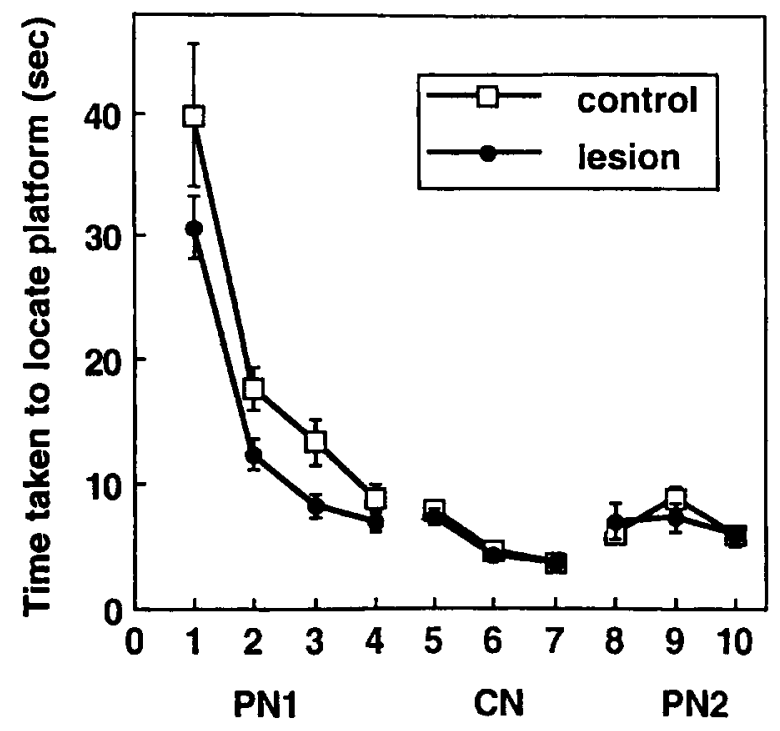

Figure 4. Mean escape latencies for control and lesioned animals during Place Navigation 1 (PN1), cued navigation (CN), and Place Navigation 2 (PN2) conditions in the Morris water maze. The performance of lesioned animals was significantly better than that of controls during PN1, but their performance during CN and PN2 was comparable to that of control animals. (Each data point represents the mean of eight trials per day.)

groups of animals spent significantly more time in the correct (southeast) quadrant during Probe Trial $2(p<$ .05 ). No statistically significant differences between groups in the number of platform crossings made during this probe trial were observed.

Place Navigation 2 in the water maze. No significant differences in escape latency between control and lesioned rats were found during PN2. Similarly, control and perirhinal lesioned animals performed equally well in Probe Trial 3-an ANOVA revealed a significant quadrant effect for both groups of animals $[F(3,75)=$ $118, p<.0001]$ but no between-group differences. Post hoc analyses demonstrated that both groups of subjects spent significantly more time swimming in the quadrant (southeast) that had previously contained the platform $(p<.05)$. There were no significant differences in the number of platform crossings made by control and lesioned animals during this probe trial.

\section{DISCUSSION}

The results of the present study indicate that subtotal damage to the superficial layers of the perirhinal cortex is associated with an increase in exploratory behavior and an increase in rearing. The increased exploratory activity was exhibited as a greater tendency for rats to revisit both neutral and novel stimuli during the course of the experiment, and the increased rearing was evident in the perirhinal lesioned rats' tendency to rear both more frequently and for a longer duration. Grooming and locomotor activity did not appear to be affected by the lesions.

Although a directly comparable study has not been conducted on animals with hippocampal damage, it has been demonstrated previously that hippocampectomized rats generally show an increase in ambulatory or locomotor activity over that of control animals. Rearing appears to be less consistently affected by hippocampal lesions, with some studies showing an increase in rearing, and others, a decrease (for a review, see Gray \& McNaughton, 1983). Interestingly, it has also been reported that exploratory activity (sniffing, biting, rearing) in both the home cage and in a novel situation is increased following hippocampal lesions (Jarrard, 1968). In contrast, entorhinal cortex lesions result in decreased exploratory activity as measured by the amount of time spent in exploring novel objects (Kohler \& Sundberg, 1977). These researchers also found that rearing and locomotor activities were increased while grooming was decreased. Similarly, Myhrer $(1988,1989)$ found that lesions which disrupted connections between the entorhinal cortex and either the hippocampus or temporal cortex resulted in a marked decrease in the lesioned animals' preference for novelty. Damage to these regions also resulted in increased rearing activity and in decreased grooming.

Rats with bilateral damage to the amygdala exhibit more rearing, sniffing, and walking behavior than do control rats (White \& Weingarten,1976). The effects are similar for subtotal amygdala lesions. For example, damage to the basolateral, lateral, or central nuclei also produce an increase in exploratory activity and reactivity to novelty in the open field test . Interestingly, amygdala lesions do not produce deficits on spatial tasks such as

Table 2

Effects of Lesions of the Perirhinal Cortex, Perforant Path, Entorhinal Cortex, Hippocampus, and Amygdala

\begin{tabular}{|c|c|c|c|c|c|}
\hline Behavior & Perirhinal & $\begin{array}{l}\text { Perforant } \\
\text { Path }\end{array}$ & $\begin{array}{l}\text { Temporal/ } \\
\text { Entorhinal }\end{array}$ & Hippocampus & Amygdala \\
\hline $\begin{array}{l}\text { Rearing } \\
\text { Exploration } \\
\text { Locomotor } \\
\text { Grooming } \\
\text { Spatial memory }\end{array}$ & $\begin{array}{l}\text { increased } \\
\text { increased } \\
\text { no change } \\
\text { no change } \\
\text { no change }\end{array}$ & $\begin{array}{l}\text { increased }^{\mathrm{a}} \\
\text { decreased }^{\mathrm{a}} \\
\text { no change }^{\mathrm{a}} \\
\text { decreased }^{\mathrm{a}} \\
\text { impaired }^{\mathrm{b}}\end{array}$ & $\begin{array}{l}\text { increased }^{c} \\
\text { decreased }^{\mathfrak{c}} \\
\text { increased }^{\mathfrak{c}} \\
\text { no change }^{\mathfrak{c}} \\
\text { impaired }^{\mathrm{d}}\end{array}$ & $\begin{array}{l}\text { varied }^{\mathrm{e}, \mathrm{f}} \\
\text { increased }^{\mathrm{g}} \\
\text { increased }^{\mathrm{g}, \mathrm{h}} \\
\text { impaired }^{\mathrm{i}}\end{array}$ & $\begin{array}{l}\text { increased }^{\mathrm{j}} \\
\text { increased }^{\mathrm{j}, \mathrm{k}} \\
\text { increased }^{\mathrm{j}, \mathrm{k}} \\
\text { no change }^{\mathrm{j}} \\
\text { no change }^{\mathrm{l}}\end{array}$ \\
\hline
\end{tabular}

${ }^{a}$ Myhrer (1988). bSkelton \& McNamara (1992). ${ }^{c}$ Myhrer (1989). ${ }^{d}$ Schenk and Morris (1985). ${ }^{\text {e}}$ Capobianco, MacDonald, \& Foster (1977). f Murphy, Race, and Brown (1975). gJarrard (1968). hTeitelbaum and Milner (1963). iMorris, Garrud, Rawlins, \& O'Keefe (1982), jWhite \& Weingarten (1976). k'Lorenzini, Baldi, Bucherelli, Giachetti, \& Tassoni (1991). 'Sutherland \& MacDonald (1990). 
the Morris water maze (Sutherland \& McDonald, 1990). Although a direct comparison between these various studies is difficult because of differences in both methodology and the nature and extent of the lesions, there is some indication that subtotal perirhinal lesions may produce a mixture of the behavioral syndromes associated with damage to other limbic structures, with the closest approximation being to lesions of the amygdaloid nuclei (Table 2). These results suggest that lesions of the superficial perirhinal cortex may, via their disruption of afferent and/or efferent flow between the amygdala and other cortical or limbic structures, produce a behavioral profile similar to that observed in animals with damage to the amygdaloid complex.

It has recently been determined that lesions of the primate perirhinal and parahippocampal cortices, or perirhinal and entorhinal cortices, result in severe memory deficits (Gaffan \& Murray, 1992; Zola-Morgan, Squire, Alvarez-Royo, \& Clower, 1991; Zola-Morgan et al., 1989). The finding that the memory deficit following these cortical lesions was, in fact, more severe than that found after hippocampal lesions alone suggests that, in primates, the perirhinal and parahippocampal cortices may make their own significant contribution to normal memory function (Squire, 1992; Zola-Morgan et al., 1993). We have also recently determined that more extensive perirhinal lesions produce a deficit in water maze performance in the rat (Wiig \& Bilkey, 1993). In the present study, subtotal lesions of superficial perirhinal cortex did not decrease water maze performance. This finding suggests that the circuitry which is critical for spatial reference memory is left intact after the lesioning procedure utilized in this study.

It has been calculated that approximately $25 \%$ of the perirhinal cortex was damaged by the lesioning procedure utilized in the present study. This percentage is based on the assumption that the borders of perirhinal cortex are accurately described by Deacon et al. (1983). Alternative descriptions of the perirhinal cortex exist, however, and one consequence of adopting an alternative definition would be an alteration in the lesion calculations. Although this might alter the percentage figure, it should be stressed that Deacon et al.'s (1983) description is relatively conservative, and that the lesions produced in the present study would be inside the boundaries of the structure if alternative definitions were adopted.

It is interesting that in the present study latencies to escape to the hidden platform were significantly shorter for perirhinal lesioned animals than for control animals during the initial place navigation task ( $\mathrm{PN} 1$; although it should be stressed that the probe trial measures did not indicate a difference in performance between groups). The mechanisms underlying this slight performance improvement are unclear, although it does not appear to have been due to differences in swimming speed. One could speculate that the increase in exploratory behavior exhibited by lesioned rats in other tasks may have had functional consequences in the water maze. If lesioned rats "sampled the environment" more often (as evidenced by increased exploration in other tasks) during the water maze procedure, they may have been able to determine the relationship between spatial cues more rapidly and therefore may have been more adept at the water maze problem.

In conclusion, the present study demonstrates that subtotal bilateral damage to the superficial perirhinal cortex in the rat results in a unique syndrome, incorporating a subset of the behavioral symptoms observed following lesions of other limbic system structures. Further research should help to clarify the role of the perirhinal cortex with regard to its relationships to these structures and provide further insight into the functional connectivity of this system.

\section{REFERENCES}

BLACKSTAD, T. W. (1958). On the termination of some afferents to the hippocampus and fascia dentata. Acta Anatomy, 35, 202-214.

Capobianco, S., MacDougall, J. M., \& Foster, S. M. (1977). Direct neurobehavioral comparisons within the septohippocampal system. Physiological Psychology, 5, 215-220.

Deacon, T. W., Eichenbaum, H., Rosenberg, P., \& Eckmann, K. W. (1983). Afferent connections of the perirhinal cortex in the rat. Journal of Comparative Neurology, 220, 168-190.

GaFFAN, D., \& MuRRAY, E. A. (1992). Monkeys with rhinal cortex ablations succeed in object discrimination learning despite $24-\mathrm{hr}$ intervals and fail at matching to sample despite double sample presentations. Behavioral Neuroscience, 106, 30-38.

GRAY, J. A., \& MCNaughton, N. (1983). Comparison between the behavioral effects of septal and hippocampal lesions: A review. Neuroscience \& Biobehavioral Reviews, 7, 119-188.

HJORTH-Simonsen, A. (1972). Projection of the lateral part of the entorhinal area to the hippocampus and fascia dentata. Journal of Comparative Neurology, 146, 219-232.

Insauti, R., AmaRaL, D. G., \& Cowan, W. M. (1987). The entorhinal cortex of the monkey: II. Cortical afferents. Journal of Comparative Neurology, 264, 356-395.

JARRARD, L. E. (1968). Behavior of hippocampal lesioned rats in home cage and novel situations. Physiology \& Behavior, 3, 65-70.

KoHLER, C., \& SUNDBERG, H. (1977). Locomotor activity and exploratory behavior after medial entorhinal cortex lesions in the albino rat. Behavioral Biology, 20, 419-432.

Kosel, K. C., van Hoesen, G. W., \& Rosene, D. L. (1983). A direct projection from the perirhinal cortex (area 35) to the subiculum in the rat. Brain Research, 269, 347-351.

Lorenzini, C. A., Baldi, E., Bucherelli, C., Giachetti, A., \& TAssonI, G. (1991). Effects of nucleus basolateralis amygdalae neurotoxic lesions on some spontaneous activities in the rat. Physiology \& Behavior, 50, 1215-1219.

Morris, R. G. M., Garrud, P., Rawlins, J. N. P., \& O'Keefe, J. (1982). Place navigation impaired in rats with hippocampal lesions. Nature, 297, 681-683.

MuRPhY, L. R., RACE, K. E., \& Brown, T. S. (1975). Behaviors emitted by rats with limbic lesions during feeding. Behavioral Biology, 15, 231-237.

MurRay, E. A., \& Mishxin, M. (1986). Visual recognition in monkeys following rhinal cortical ablations combined with either amygdalectomy or hippocampectomy. Journal of Neuroscience, $\mathbf{6}$, 1992-2003.

MyHRER, T. (1988). Exploratory behavior and reaction to novelty in rats with hippocampal perforant path systems disrupted. Behavioral Neuroscience, 102, 356-362.

MyHRER, T. (1989). Exploratory behavior, reaction to novelty, and proactive memory in rats with temporo-entorhinal connections disrupted. Physiology \& Behavior, 45, 431-436. 
Otto, T., \& Eichenbaum, H. (1992). Complementary roles of the orbital prefrontal cortex and the perirhinal-entorhinal cortices in an odor-guided delayed-nonmatching-to-sample task. Behavioral Neuroscience, 106, 762-775.

PAXINOS, G., \& WATSON, C. (1982). The rat brain in stereotaxic coordinates. Sydney: Academic Press.

SAunders, R. C., \& Rosene, D. L. (1988). A comparison of the efferents of the amygdala and the hippocampal formation in the rhesus monkey: I. Convergence in the entorhinal, prorhinal and perirhinal cortices. Journal of Comparative Neurology, 271, 153-184.

SCHENK, F., \& MoRRIs, R. G. M. (1985). Dissociation between components of spatial memory after recovery from the effects of retrohippocampal lesions. Experimental Brain Research, 58, 11-28.

Skelton, R. W., \& McNamaRA, R. K. (1992). Bilateral knife cuts to the perforant path disrupt spatial learning in the Morris water maze. Hippocampus, 2, 73-80.

Souire, L. R. (1992). Memory and the hippocampus: A synthesis from findings with rats, monkeys and humans. Psychological Review, 99, 195-231.

Sutherland, R. J., \& MCDonald, R. J. (1990). Hippocampus, amygdala and memory deficits in rats. Behavioral Brain Research, 37, 57-79.

Suzuki, W. A., \& Amaral, D. G. (1990). Cortical inputs to the CA1 field of the monkey hippocampus originate from the perirhinal and parahippocampal cortex but not from area TE. Neuroscience Letters, 115, 43-48.

Suzuki, W. A., Zola-Morgan, S., SQuire, L. R., \& Amaral, D. G. (1993). Lesions of the perirhinal and parahippocampal cortices in the monkey produce long lasting memory impairment in the visual and tactile modalities. Journal of Neuroscience, 13, 2430-2451.
Teitelbaum, H., \& Milner, P. (1963). Activity changes following partial hippocampal lesions in rats. Journal of Comparative \& Physiological Psychology, 56, 284-289.

WhITE, N., \& WEINGARTEN, H. (1976). Effects of amygdaloid lesions on exploration by rats. Physiology and Behavior, 17, 73-79.

WiIG, K. A., \& BILKEY, D. K. (1993, August). The effects of perirhinal cortical lesions on spatial reference memory in the rat. Paper presented at the International Australasian Winter Conference on Brain Research, Queenstown, New Zealand.

Witter, M. P., Groenewegen, H. J., Lopes da Silva, F. H., \& LoHMaN, A. H. M. (1989). Functional organization of the extrinsic and intrinsic circuitry of the parahippocampal region. Progress in Neurobiology, 33, 161-253.

ZiLLES, K. (1990). Anatomy of the neocortex: Cytoarchitecture and myeloarchitecture. In B. Kolb \& R. C. Tees (Eds.), The cerebral cortex of the rat (pp. 77-112). Cambridge, MA: MIT Press.

Zola-Morgan, S., Squire, L. R., Alvarez-Royo, P., \& Clower, R. P. (1991). Independence of memory functions and emotional behavior: Separate contributions of the hippocampal formation and the amygdala. Hippocampus, 1, 207-220.

Zola-Morgan, S., Squire, L. R., Amaral, D. G., \& Suzuki, W. (1989). Lesions of the perirhinal and parahippocampal cortex that spare the amygdala and the hippocampal formation produce severe memory impairment. Journal of Neuroscience, 9, 4335-4370.

Zola-Morgan, S., SQuire, L. R., Clower, R. P., \& RemPel, N. L. (1993). Damage to the perirhinal cortex exacerbates memory impairment following lesions to the hippocampal formation. Journal of Neuroscience, 13, 251-265.

(Manuscript received August 2, 1993; revision accepted for publication January 11,1994 .) 